\title{
Accurate measurement of tip-sample contact size during nanoindentation of viscoelastic materials
}

\author{
B. Tang and A.H.W. Ngan \\ Department of Mechanical Engineering, The University of Hong Kong, Pokfulam Road, \\ Hong Kong, People's Republic of China
}

(Received 5 November 2002; accepted 6 February 2003)

\begin{abstract}
Polypropylene (PP) and amorphous selenium ( $a$-Se) were used as prototype materials at room temperature to explore the problems that may exist in the accurate measurement of the reduced modulus of viscoelastic materials using depth-sensing nanoindentation. As has been reported previously by others, we observed that a "nose" in the load-displacement curve may occur during unloading, indicating significant creep effects at the onset of unloading. To accurately measure the elastic modulus in viscoelastic materials like PP or $a$-Se, both the contact stiffness and the contact area at the onset of unloading must be determined accurately. The issue of removing the influence of creep on the measurement of the contact stiffness using the Oliver-Pharr method has been addressed in a previous paper by Feng and Ngan. In this work, the effect of creep on contact-depth measurement is considered. Removal of creep effects in both contact stiffness and contact-area measurement leads to satisfactory prediction of the reduced moduli in PP and $a$-Se.
\end{abstract}

\section{INTRODUCTION}

A decade ago, Oliver and Pharr ${ }^{1}$ proposed a novel method to determine the hardness and elastic modulus of a material using depth-sensing indentation. The OliverPharr method has since become a standard method in the analysis software of commercially available nanoindenters supplied by, for example, MTS, Hysitron, CSM Instruments SA, MicroMaterials, etc. In the Oliver-Pharr scheme, it is assumed that, during the unloading process, the contact between the tip and the surface is purely elastic. The result is the well-known formula for obtaining the contact depth $h_{\mathrm{c}}$ :

$$
h_{\mathrm{c}}=h_{\max }-\epsilon \frac{P_{\max }}{S},
$$

where $h_{\max }$ is the maximum indenter displacement at the onset of unloading, $P_{\max }$ is the load before unloading, $S$ is the contact stiffness at the onset of unloading, and $\epsilon$ is a constant ( $\epsilon=0.75$ for Berkovich tip). $S$ is measured by fitting the load $(P)$ versus displacement $(h)$ curve during unloading to the empirical equation $P=a\left(h-h_{\mathrm{f}}\right)^{m}$, where $a, h_{\mathrm{f}}$, and $m$ are fitting parameters. ${ }^{1,2}$ Once the contact depth $h_{\mathrm{c}}$ is calculated from Eq. (1), the contact area $A_{\mathrm{c}}$ can be obtained from the known shape function of the indenter tip, and finally, the reduced modulus, $E_{\mathrm{r}}$ can be obtained from the classical contact mechanics result

$$
E_{\mathrm{r}}=\frac{\sqrt{\pi}}{2} \frac{S}{\sqrt{A_{\mathrm{c}}}} .
$$

Both Eq. (1) and (2) are based on the assumption that the tip-sample contact is purely elastic.

Unfortunately, in many cases, the contact between the tip and the sample is far from purely elastic. Creep effects during indentation have been reported by many researchers. ${ }^{3-17}$ It is well known that creep effects during unloading may cause the contact stiffness to be overestimated..$^{3,4,8}$ In extreme cases of viscosity becoming the dominant factor during unloading, as would happen when the unloading rate is low or the hold before unloading is too short or the full load is large enough, the indenter displacement may continue to increase for a short while during the initial stage of unloading. In this situation, a "nose" will appear in the corresponding loaddisplacement curve. ${ }^{3,8,16,17}$ Figure 4 will show an example. When a nose occurs, the apparent unloading stiffness will be negative, but even when a conspicuous nose does not occur, the apparent stiffness may still be a severe overestimation of the true elastic contact stiffness. In this case, the elastic modulus calculated by the OliverPharr method may become very unreliable. 
In a recent investigation, Feng and $\mathrm{Ngan}^{3,4}$ proposed a simple method to correct for the measurement of contact stiffness $S$. Assuming linear viscoelasticity, they showed that, in an experiment involving a brief load hold prior to unloading, the relationship between the true (elastic) unloading stiffness $S_{\mathrm{e}}$ and the observed unloading stiffness $S$ is given by

$$
\frac{1}{S_{\mathrm{e}}}=\frac{1}{S}+\frac{\dot{h}_{\mathrm{h}}}{|\dot{P}|}
$$

where $\dot{P}$ is the unloading rate, and $\dot{h}_{\mathrm{h}}$ is the displacement rate (the creep rate) just prior to unloading. $\dot{h}_{\mathrm{h}}$ can be calculated by fitting the $h(t)$ curve ( $t=$ time) during the load hold by certain model curves. Feng and Ngan showed that once the $S_{\mathrm{e}}$ is used for $S$ in Eq. (2), accurate reduced moduli can be obtained in metallic materials including $\mathrm{Cu}, \mathrm{Al}$, and $\mathrm{Ni}_{3} \mathrm{Al}$.

However, Feng and Ngan's procedure leaves the issue of $A_{\mathrm{c}}$ unaddressed. The spirit of Oliver-Pharr's Eq. (1) is to use the unloading curve to infer the contact depth at the onset of unloading. Figure 1 shows the accuracy of Eq. (1) to different extents of viscosity during unloading, caused by using different unloading rates, in polypropylene (PP) at room temperature. In these experiments, the load schedules prior to unloading were all kept constant, but the unloading rate was varied (Fig. 2). The actual contact size at the onset of unloading should therefore be the same in all the experiments. However, Fig. 1 indicates clearly that the $h_{\mathrm{c}}$ results calculated by Eq. (1) increased sharply with decreasing unloading rate. For instance, when the unloading rate was $1600 \mathrm{mN} / \mathrm{min}$, the contact depth was $9342 \mathrm{~nm}$, but when the unloading rate became $20 \mathrm{mN} / \mathrm{min}$, the calculated $h_{\mathrm{c}}$ increased to $14,729 \mathrm{~nm}$.

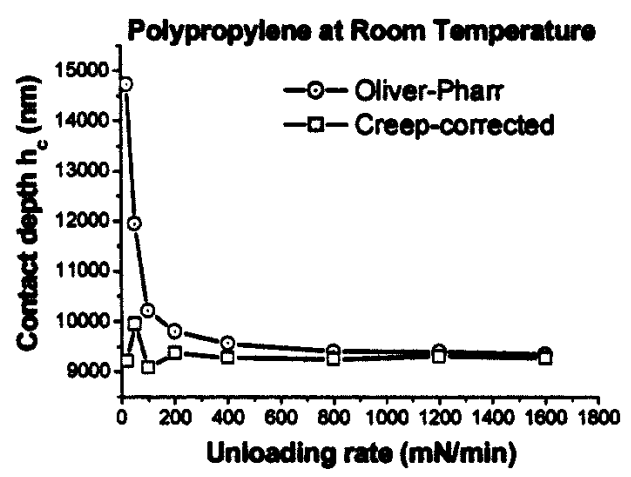

FIG. 1. Effect of unloading rate on the contact depth calculated by the Oliver-Pharr method and that calculated by Eq. (8) in the text. Data from different indentation experiments are employing the same loading rate of $800 \mathrm{mN} / \mathrm{min}$, peak load of $200 \mathrm{mN}$, and holding time of $30 \mathrm{~s}$ at the peak load (see Fig. 2). The unloading rates are, however, different as shown. The contact depth calculated by Eq. (8) tends to be a constant value, which seems reasonable.
In this paper, a simple procedure to correct the contact depth for creep effects is proposed. To validate our approach, we will use PP and amorphous selenium $(a-\mathrm{Se})$ as prototype viscoelastic materials. Since PP and $a$-Se creep much more severely than the metals used by Feng and Ngan, their proposed formula for fitting the creep curve was found to be unsuitable for PP and $a$-Se. A new fitting formula for very soft materials is also proposed here. We believe that the viscoelastic behavior of PP and $a$-Se at room temperature is prototypic to that of materials with higher melting points at high temperatures, so the present results should be useful to the development of high-temperature nanoindentation techniques that are in progress worldwide.

Since this study focused on the applicability of a creep correction procedure, we have made no attempt to characterize the microstructural changes during indentation. In other words, the materials used here are treated here as "black boxes" in terms of microstructure. We cannot rule out the occurrence of, for example, stress-induced crystallization under the high pressures during nanoindentation. However, insofar as our concern is elasticity, any microstructural changes within the small material volume in the indent core should not have significant effects on the measured tip-sample contact stiffness.

\section{MODIFIED PROCEDURE FOR CONTACT DEPTH CALCULATION}

Here, we develop a simple formula that can be used to correct for the creep effects in the contact depth $h_{\mathrm{c}}$. We first assume that the total displacement $h$ is the sum of (i) an elastic displacement $h_{\mathrm{e}}$, (ii) a time-dependent creep displacement $h_{\mathrm{t}}$, and (iii) a plastic displacement $h_{\mathrm{f}}$; i.e.,

$$
h=h_{\mathrm{e}}+h_{\mathrm{t}}+h_{\mathrm{f}}
$$

We then assume that Sneddon's result ${ }^{18}$ for conical indentation onto an elastic half-space, $h_{\mathrm{c}} / h=2 / \pi$, holds

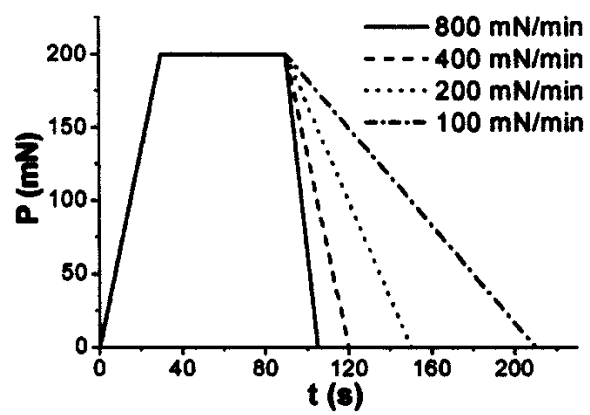

FIG. 2. Loading schedules used to produce the response shown in Fig. 1. The holding time and loading rate were the same, but unloading rates were different. 
for the elastic component only. Thus from Eq. (4), we have

$$
\frac{h_{\mathrm{c}}-h_{\mathrm{f}}-h_{\mathrm{t}}}{h-h_{\mathrm{f}}-h_{\mathrm{t}}}=\frac{2}{\pi}
$$

which can be rearranged into

$h_{\mathrm{c}}=h-\left(1-\frac{2}{\pi}\right)\left(h-h_{\mathrm{f}}-h_{\mathrm{t}}\right)=h-\left(1-\frac{2}{\pi}\right) h_{\mathrm{e}}$.

The development up to this stage is similar to that used by Oliver and Pharr ${ }^{1}$ in deriving Eq. (1); the only difference is the addition of the creep term $h_{\mathrm{t}}$. It should be noted, however, that the decomposition of the total displacement into the elastic, plastic, and creep components in Eq. (4) can at best be treated as a phenomenological approximation only. Even for the case of linear viscoelasticity, the exact analysis by Ngan and Feng ${ }^{3}$ indicates that the total displacement is not the sum of the elastic and viscous components. A full solution of the elastoviscoplastic indentation problem can only be obtained numerically, but the approximation in Eq. (4) enables an approximate, analytical solution to be found. The justification of the approximation involved here can only be sought from the ability of the approach to satisfactorily correct for creep in the elastic modulus measurement, which will be dealt with in the later sections of this paper.

With Eq. (4), the contact stiffness at the onset of unloading $S$ is given by

$\frac{1}{S}=\frac{\mathrm{d} h}{\mathrm{~d} P}=\frac{\mathrm{d}\left(h_{\mathrm{e}}+h_{\mathrm{f}}+h_{\mathrm{t}}\right)}{\mathrm{d} P}=\frac{\mathrm{d} h_{\mathrm{e}}}{\mathrm{d} P}+\frac{\mathrm{d} h_{\mathrm{t}}}{\mathrm{d} P}=\frac{\mathrm{d} h_{\mathrm{e}}}{\mathrm{d} P}+\frac{\dot{h}_{\mathrm{h}}}{\dot{P}}$.

In the last step in Eq. (6), we have, as in Feng and Ngan, ${ }^{3,4}$ used the condition that the creep rate at the onset of unloading is identical to that at the end of the load hold $\dot{h}_{\mathrm{h}}$. As in Oliver and Pharr, ${ }^{1}$ we further assume that $P \propto h_{\mathrm{e}}{ }^{2}$ during unloading, and hence $\mathrm{d} P / P=2 \mathrm{~d} h_{\mathrm{e}} / h_{\mathrm{e}}$. Hence, from Eq. (6),

$h_{\mathrm{e}}=2 P \frac{\mathrm{d} h_{\mathrm{e}}}{\mathrm{d} P}=2 P\left(\frac{1}{S}-\frac{\dot{h}_{\mathrm{h}}}{\dot{P}}\right)=\frac{2 P}{S}\left(1+S \frac{\dot{h}_{\mathrm{h}}}{|\dot{P}|}\right)$.

Substituting Eq. (7) into Eq. (5) yields

$$
h_{\mathrm{c}}=h-\epsilon \frac{P}{S}\left(1+S \frac{\dot{h}_{\mathrm{h}}}{|\dot{P}|}\right)=h-\epsilon \frac{P}{S_{\mathrm{e}}},
$$

where $\epsilon=2(1-2 / \pi)$ is the same constant as in Oliver and Pharr's result in Eq. (1), and $S_{\mathrm{e}}$ is the same corrected stiffness as given by Feng and Ngan's result in Eq. (3). The correction formula for the contact depth in Eq. (8) therefore has the same form as Oliver and Pharr's original Eq. (1), except that the contact stiffness has to be corrected for creep using Feng and Ngan's Eq. (3) above.

\section{EXPERIMENTAL}

\section{A. Experimental procedures}

All indentation experiments reported here were carried out on PP and $a$-Se. The PP sample was prepared by injection molding and its specifications are as follows: (i) degree of crystallinity 53\%; (ii) melting point $169^{\circ} \mathrm{C}$; (iii) glass transition temperature $-3{ }^{\circ} \mathrm{C}$; (iv) density 0.9 $0.91 \mathrm{~g} / \mathrm{cm}^{3}$. The PP sample was in the form of a slab with dimensions of about $2 \mathrm{~cm} \times 2 \mathrm{~cm} \times 3 \mathrm{~mm}$ and was polished down to about $1 \mu \mathrm{m}$ in roughness before indentation. The $a$-Se sample with a smooth surface was prepared by first melting a $99.99 \%$ pure selenium button at $300{ }^{\circ} \mathrm{C}$ and then quenching to room temperature to avoid crystallization. The indentation experiments were carried out on a nanoindenter supplied by CSM Instruments SA in Switzerland. The probe used was a Berkovich tip (CSM Instrument SA, Peseux, Switzerland). Load schedules employed typically comprised first a constant-rate load ramp to the desired peak load, followed by holding at the peak load for a selected duration and then constant-rate unloading. In some experiments, a stepwise increasing load schedule as shown in Fig. 3 was also used. The purpose of using this load schedule is to vary the creep effects at the same position on the sample surface, since it has been shown previously ${ }^{17}$ that indentation creep will become more severe when the hold load increases.

Our nanoindenter has a maximum load of $300 \mathrm{mN}$, and the typical thermal drift rate is about $0.1 \mathrm{~nm} / \mathrm{s}$. To minimize surface variability and effects of thermal drift, large loads approaching the machine limit were used. All the experiments were completed in less than a few hours, so that the maximum thermal drift is only a fraction of a micron. This is much smaller than the displacement at

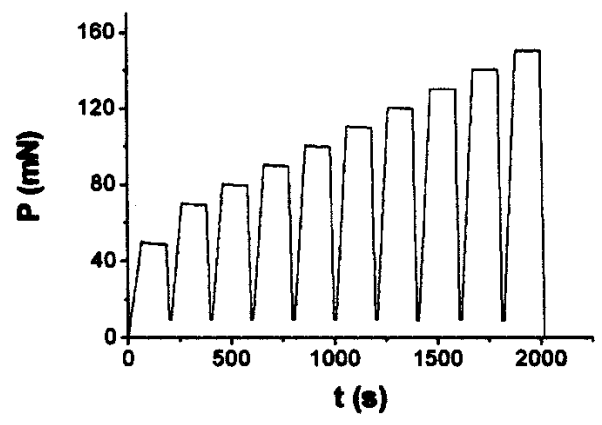

FIG. 3. Typical stepwise increasing load schedule. Since viscoelastic effects are more significant at higher hold loads, this loading schedule can be use to explore different viscoelastic effects on a reduced modulus measurement at the same position on the sample. 
peak loads, which is typically above $10 \mu \mathrm{m}$, and therefore, there was no need to perform thermal drift correction. Our machine also has a sapphire ring contacting the specimen during indentation. The machine measures the relative displacement between the tip and the ring instead of the absolute displacement of the tip alone, which is subject to influence from thermal drift, and load frame and mounting compliances.

\section{B. Data analysis procedures}

Recent experiments by the present authors ${ }^{17}$ have shown that a nose may appear in the unloading loaddisplacement curve if (i) the hold time at full load is short, (ii) the unloading rate is small, and (iii) the full load is high enough. When a nose occurs, the unloading curve can no longer be fitted accurately by the power-law formula $P=A\left(h-h_{\mathrm{f}}\right)^{m}$ proposed by Oliver and Pharr; an example of this is shown in Fig. 4. The main problem is that the Oliver-Pharr formula is a $P(h)$ mapping, but
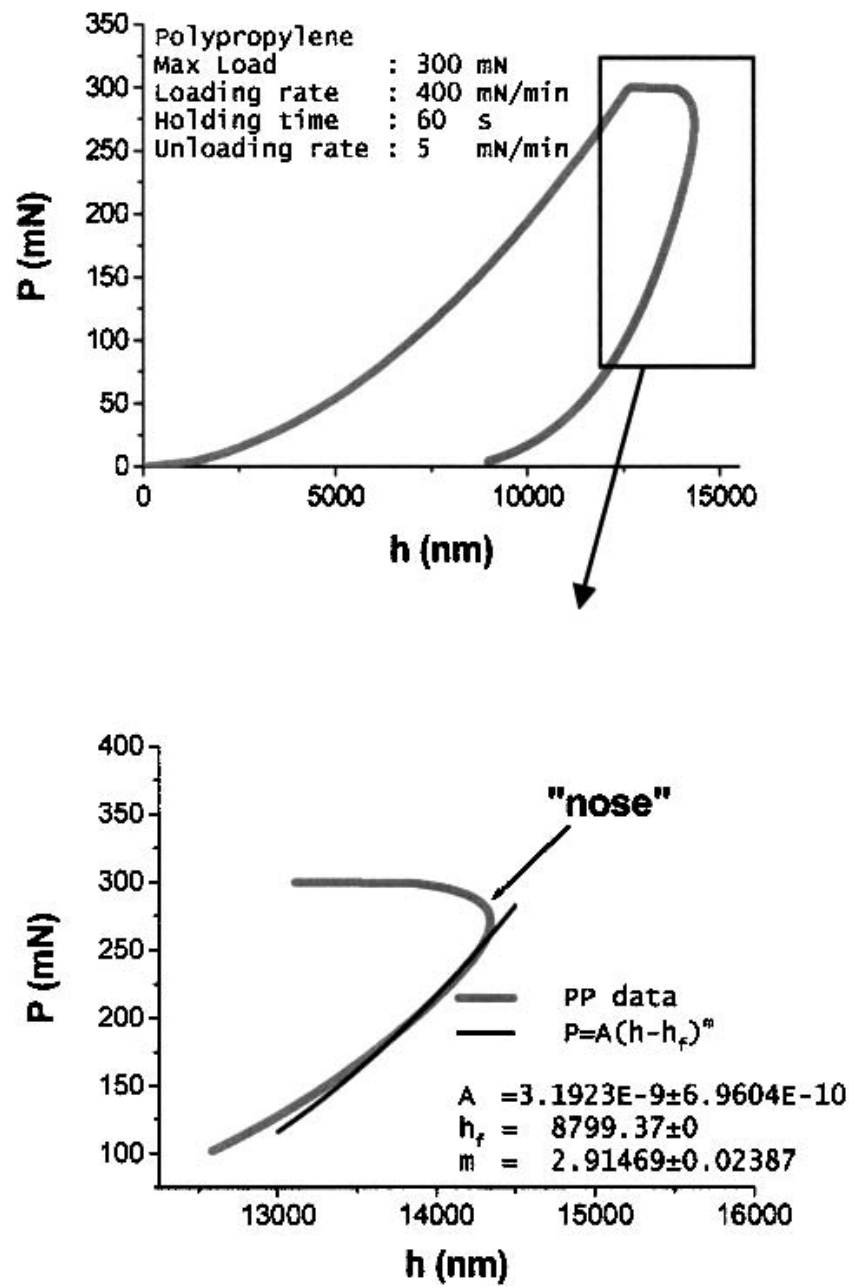

FIG. 4. A situation where a nose in the unloading curve cannot be fitted by the power-law formula proposed by Oliver and Pharr. The apparent unloading stiffness in this case is negative. when a nose occurs, one $h$ may correspond to two different values of $P$. We overcome this problem by writing the $P(h)$ mapping as $h(P)$. For a severe nose, we find the following normalized form

$y=a x^{2}+b x+c x^{1 / 2}+d x^{1 / 3}+e x^{1 / 4}+f x^{1 / 5}+g x^{1 / 6}$.

satisfactory, where $x=\left(P_{\mathrm{o}}-P\right) / P_{\mathrm{o}}, y=\left(h-h_{\mathrm{o}}\right) / h_{\mathrm{o}}$, and $a$ to $g$ are fitting parameters. The $x^{1 / 2}$ term in Eq. (9) provides the $P^{1 / 2}$ dependence in the Oliver-Pharr formula $P=A\left(h-h_{\mathrm{f}}\right)^{m}$ in which $m \sim 2$ for a conical indenter. The quadratic terms $x^{2}$ and $x$ take care of the nose shape, and the other terms are added to achieve better fit when the nose is pronounced; the fractional indices of $x$ ensure convergence and make the quality of the fit insensitive to small fluctuations in the fitting parameters $c$ to $g$. The normalization constants $P_{\mathrm{o}}$ and $h_{\mathrm{o}}$ in $x$ and $y$ are usually set to $P_{\mathrm{o}}=1.1 P_{\mathrm{u}}$ and $h_{\mathrm{o}}=0.9 h_{\mathrm{u}}$, where $P_{\mathrm{u}}$ and $h_{\mathrm{u}}$ are the load and displacement at the onset of unloading. Figure 5(a) shows that even for a severe nose, Eq. (9) can fit the unloading loaddisplacement curve very well. After all the fitting parameters in Eq. (9) are known, one can easily calculate the contact stiffness, defined as $\mathrm{d} P / \mathrm{d} h$.

On the basis of the observation of the unloading data, it was found that

$$
\begin{gathered}
h=h_{\mathrm{f}}+a P^{1 / 2}+b P^{1 / 4}+c P^{1 / 8} \\
(h \text { in nm, } P \text { in mN }),
\end{gathered}
$$

can fit the unloading load-displacement curve without nose very well. Again, the first two terms up to $P^{1 / 2}$ preserve the essence of the Oliver-Pharr formula, and the remaining terms are there to provide better fit when creep is severe. An example is shown in Fig. 5(b), in which it can be seen that the parameters $b$ and $c$ can be determined free of errors. In this paper, Eq. (9) was used to fit the unloading load-displacement curve with nose, and Eq. (10) was used to fit the unloading load-displacement curve without nose.

It was also found that

$$
\begin{gathered}
h=h_{\mathrm{i}}+a\left(t-t_{\mathrm{i}}\right)^{1 / 2}+b\left(t-t_{\mathrm{i}}\right)^{1 / 4}+c\left(t-t_{\mathrm{i}}\right)^{1 / 8} \\
(h \text { in nm, } t \text { in s }),
\end{gathered}
$$

can fit the time-displacement curve during hold very well, and therefore, load hold data were fitted by this equation in this work. Here, $\left(h_{\mathrm{i}}, t_{\mathrm{i}}\right)$ are conditions at the onset of the load hold and $a$ to $c$ are fitting parameters. The rationale of Eq. (11) is based on the exact solution of the linear viscoelastic indentation problem obtained by Feng and Ngan. ${ }^{3}$ In this work, it was shown that, for constant load, $\mathrm{d}\left(h^{2}\right) / \mathrm{d} t \propto P$ or $h \propto\left(t-t_{\mathrm{i}}\right)^{1 / 2}$ [see Eq. (10) of Ref. 3]. The additional terms in Eq. (11) serve to improve the quality of the fit. 


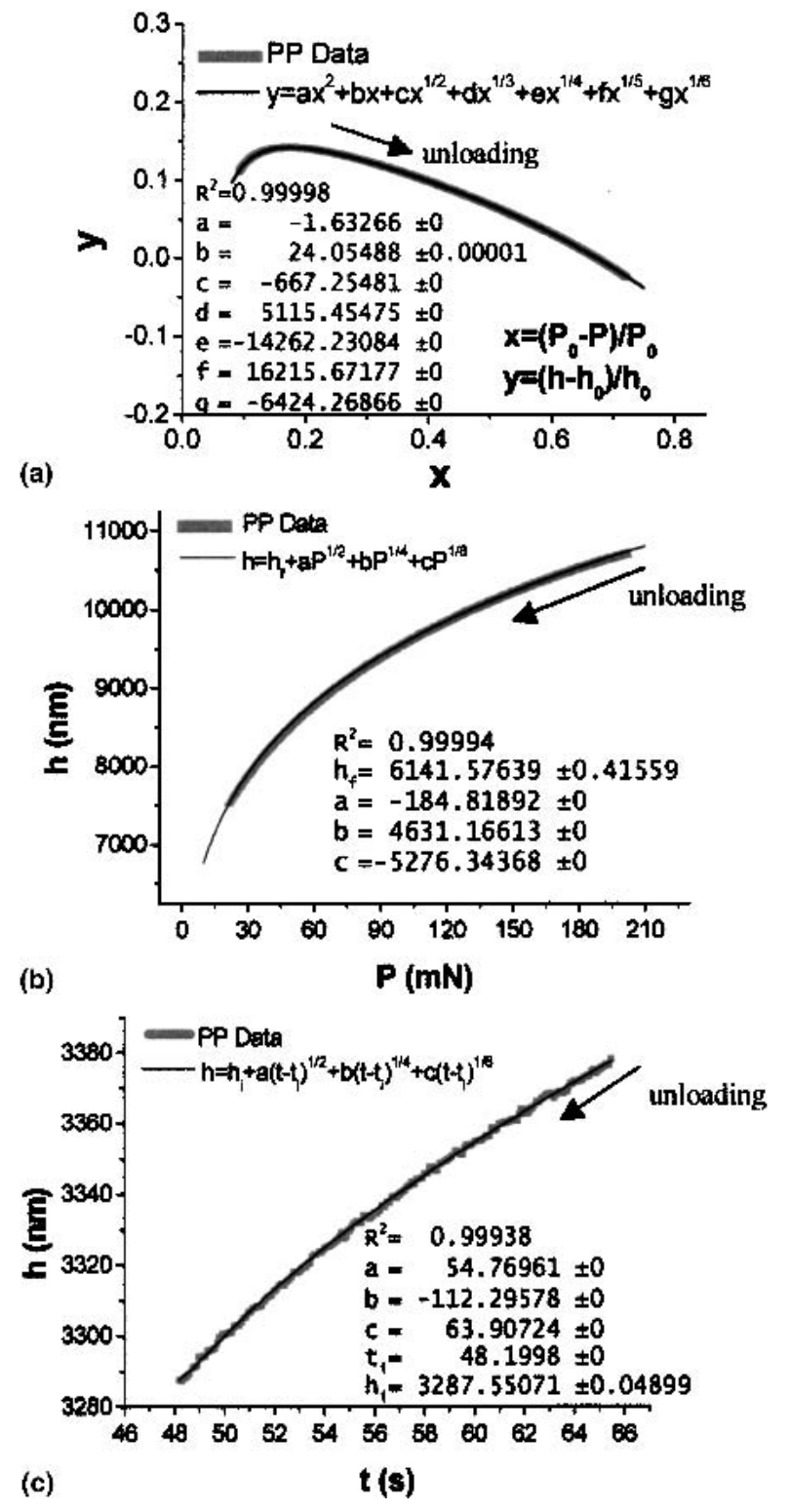

FIG. 5. Accurate fitting of (a) an unloading segment with a "nose" by Eq. (9), (b) an unloading segment without a nose by Eq. (10), and (c) a load-hold segment by Eq. (11).

\section{RESULTS AND DISCUSSION}

To investigate the validity of the method we proposed earlier, we compare indentation data from PP and $a$-Se calculated using the following three methods: (i) OliverPharr method (neither contact stiffness nor contact depth corrected for creep effects); (ii) Feng-Ngan method (only the contact stiffness corrected); (iii) the current method (both contact stiffness and contact depth corrected).

\section{A. Creep effects on $\boldsymbol{h}_{\mathrm{c}}$}

In the experiment shown in Fig. 1, the contact depth at the onset of unloading calculated by the original OliverPharr method becomes severely overestimated when the unloading rate is small. Such an overestimation is due to creep effects and can be understood by Eq. (8), which indicates that if the $\dot{h}_{\mathrm{h}} /|\dot{P}|$ term is omitted, $h_{\mathrm{c}}$ will be overestimated. Also shown in Fig. 1 are the data after correction for creep by Eq. (8). It can be seen that, after creep correction, the contact depth at the onset of unloading tends to a constant value, which makes sense as in these indentation experiments the load histories up to the point of unloading were all identical.

One thing that needs to be noted here is that the hardness, which is defined as the load defined by the contact area, may also be subject to error if the creep effects on the contact depth are not corrected.

\section{B. $\boldsymbol{S}-\boldsymbol{h}_{\mathrm{c}}$ relationship}

In this section, all the experiments employed the stepwise increasing load schedules shown in Fig. 3 and the experiment temperature was well controlled at $24 \pm 1{ }^{\circ} \mathrm{C}$. The relationships between the contact depth and the contact area calculated by the three methods mentioned above were investigated.

A perfect Berkovich tip has a shape function given by $A_{\mathrm{c}}=24.5 h_{\mathrm{c}}{ }^{2}$, and hence, from Eq. (2),

$$
\frac{h_{\mathrm{c}}}{S}=\frac{\sqrt{\pi}}{2 \sqrt{24.5} E_{\mathrm{r}}} .
$$

Figure 6 compares the shape function of the indenter tip used in this work with that of a perfect Berkovich tip. It is evident that the true tip shape can be well approximated by the ideal shape when the contact depth is over $20 \mathrm{~nm}$. Since all the contact depths during unloading presented here are over $20 \mathrm{~nm}$, it is acceptable to assume ideal tip shape and hence validity of Eq. (12). Since the experiment temperature was well controlled to be fairly constant, the reduced modulus should be constant for the

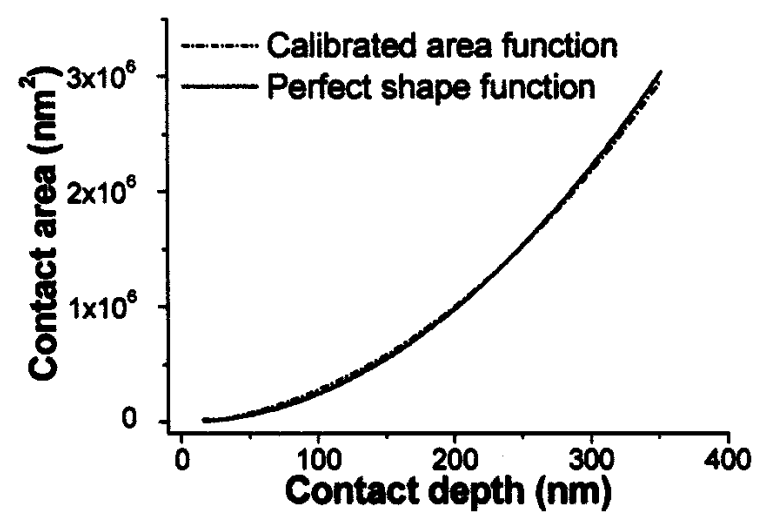

FIG. 6. Calibrated shape function of the Berkovich tip used in this work, compared against that of a perfect tip. When the contact depth is over $20 \mathrm{~nm}$, the true shape can be well approximated by the perfect tip shape. 
same specimen. Therefore, Eq. (12) predicts that the contact depth $h_{\mathrm{c}}$ should be proportional to the contact stiffness $S$.

Figure 7 shows the $h_{\mathrm{c}}$ versus $S$ plot of PP and $a$-Se with both $h_{\mathrm{c}}$ and $S$ calculated by the Oliver-Pharr method without applying creep correction. Some data
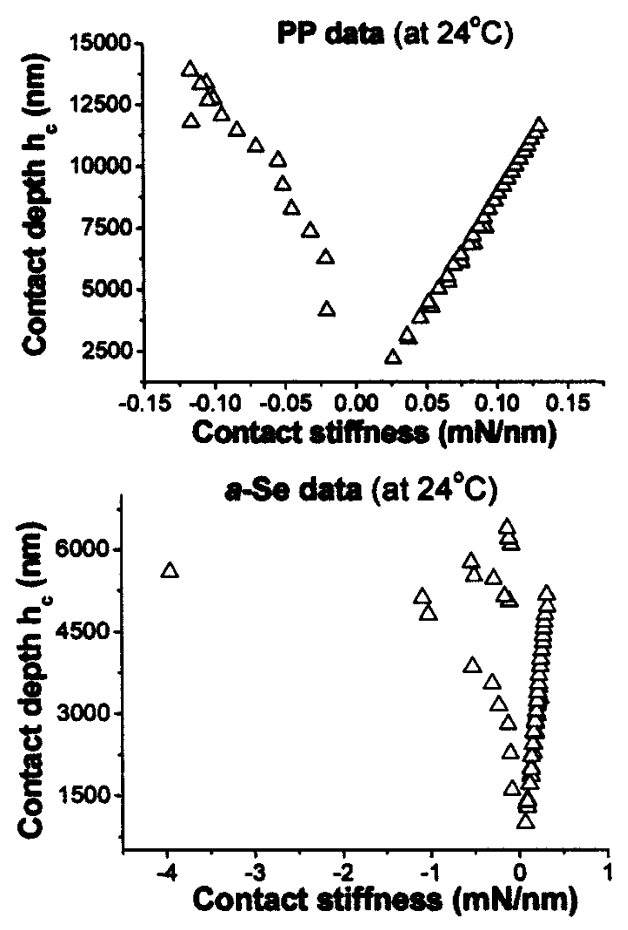

FIG. 7. Apparent contact depth versus apparent contact stiffness, calculated by the Oliver-Pharr method without creep correction.
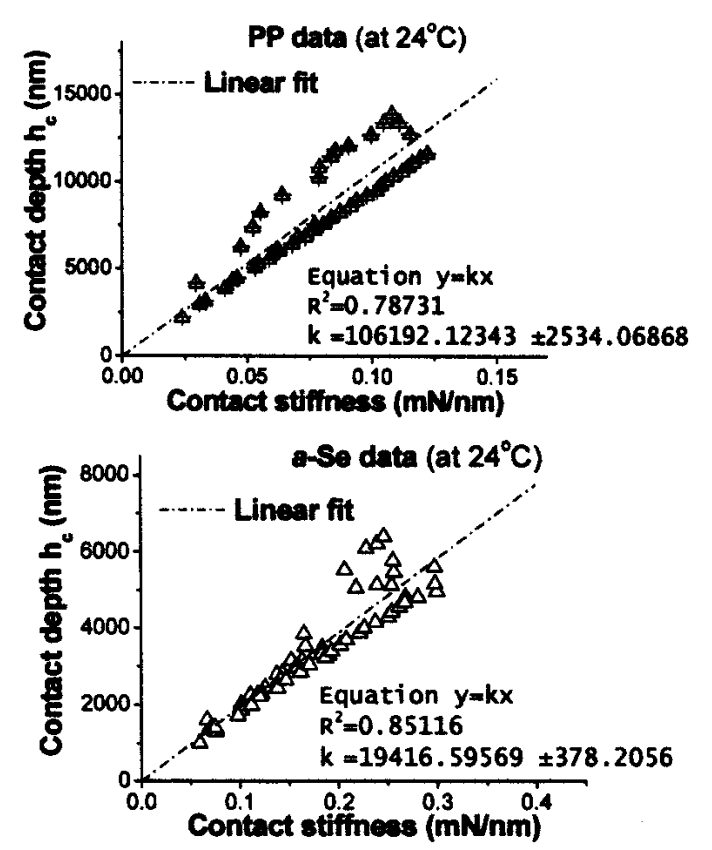

FIG. 8. Apparent contact depth (without creep correction) versus creep-corrected contact stiffness. points exhibit negative apparent contact stiffness values because of the appearance of a nose in the unloading load-displacement curves, corresponding to very severe creep effects near the onset of unloading. Obviously, the relationship between the apparent contact depth and the apparent contact stiffness is far from linear.

Figure 8 shows the plot of the apparent contact depth versus the creep-corrected contact stiffness, in which only the contact stiffness has been corrected for creep. The creep correction on the contact stiffness successfully removes the problem of negative apparent contact stiffness. However, the contact depth without creep correction does not exhibit a good proportionality relationship with the contact stiffness.

Figure 9 shows the same set of experimental data after both the contact depth and the contact stiffness have been corrected for creep by Eqs. (8) and (3), respectively. It is evident that the relationship between the corrected contact depth and contact stiffness is proportional within experimental errors. Using Eq. (12), the value of the reduced modulus $E_{\mathrm{r}}$ can be calculated from the slope of the $h_{\mathrm{c}}$ versus $S$ plot. The $E_{\mathrm{r}}$ calculated this way is $1.89 \pm 0.01 \mathrm{GPa}$ for PP and $10.3 \pm 0.1 \mathrm{GPa}$ for $a$-Se. Young's modulus can be calculated from the reduced modulus by

$$
\frac{1}{E_{\mathrm{r}}}=\frac{\left(1-v^{2}\right)}{E}+\frac{\left(1+v_{\mathrm{i}}^{2}\right)}{E_{\mathrm{i}}}
$$

where $E$ and $v$ are Young's modulus and Poisson's ratio of the sample, and the corresponding quantities of the tip are denoted by the subscript i. Poisson's ratio and
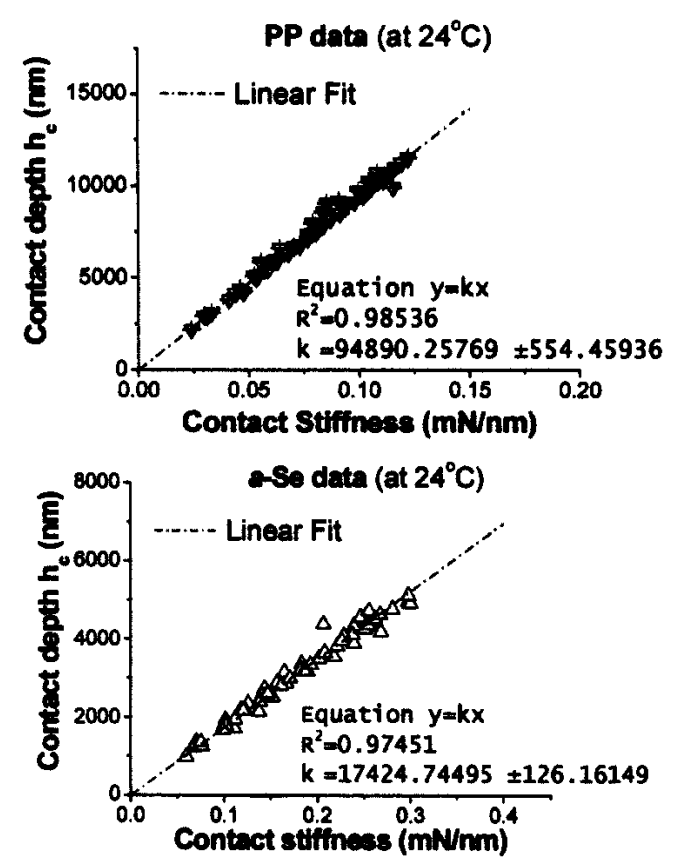

FIG. 9. Creep-correction contact depth versus creep-corrected contact stiffness. 
Young's modulus of our nanoindenter are 0.07 and $1141 \mathrm{GPa}$, respectively. Poisson's ratio of the $a-\mathrm{Se}^{19}$ is taken as 0.312 , and that of PP is assumed to be 0.4 . The calculated Young's modulus is therefore $1.59 \pm 0.01 \mathrm{GPa}$

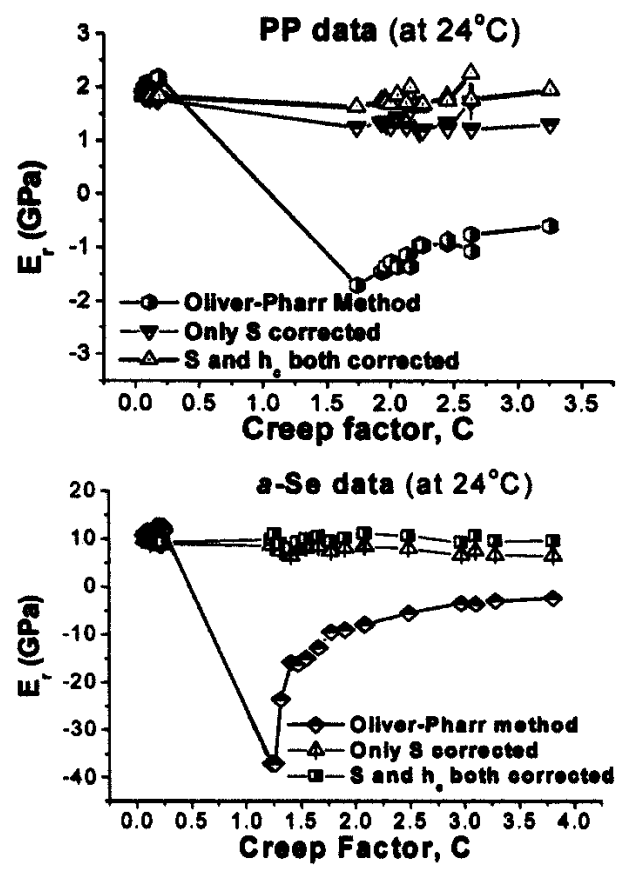

FIG. 10. Reduced modulus versus creep factor calculated by three different methods from the same experiment data at a given temperature.
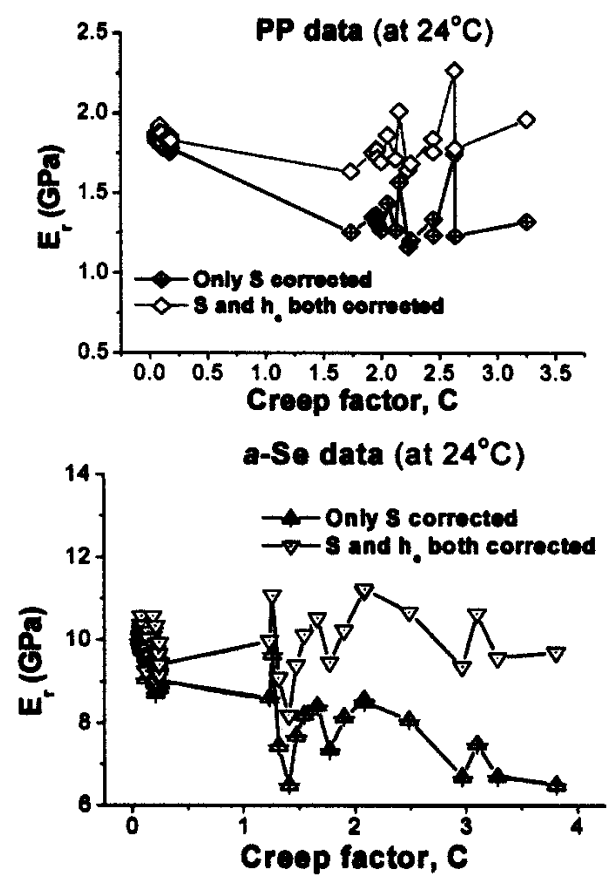

FIG. 11. Comparison between the Feng-Ngan method and the method of correcting both $S$ and $h_{\mathrm{c}}$. for PP and $9.38 \pm 0.09 \mathrm{GPa}$ for $a$-Se. These agree well with the values reported in the literature (1.2 to $1.7 \mathrm{GPa}$ for $\mathrm{PP},{ }^{20} 10 \mathrm{GPa}$ at $290 \mathrm{~K}$ to about $7 \mathrm{GPa}$ at $310 \mathrm{~K}$ for $\left.a-\mathrm{Se}^{21}\right)$.

\section{Reduced modulus}

In Feng and Ngan's work, ${ }^{3,4}$ a creep factor, defined as

$$
C=\frac{\dot{h}_{\mathrm{h}} S_{\mathrm{e}}}{|\dot{P}|}
$$

was used to represent the significance of the creep effects during depth-sensing indentation. Figure 10 shows the reduced modulus of the two materials calculated by the Oliver-Pharr scheme [Eq. (2)], the Feng-Ngan scheme, and the present scheme of correcting both $h_{\mathrm{c}}$ and $S$ for creep effects. The reduced modulus calculated using the Oliver-Pharr method becomes negative when the creep factor is large, and this, as discussed above, is due to the occurrence of a nose in the unloading loaddisplacement curve. Compared with the Oliver-Pharr scheme, both the Feng-Ngan scheme and the present scheme seem to work well, and the reduced modulus calculated by these two schemes is positive for any creep factor.

To see the effect of contact depth correction, the FengNgan scheme is further compared with the present scheme in a magnified scale in Fig. 11. It can be seen that, with increasing creep factor, PP's reduced modulus calculated by the Feng-Ngan scheme drops from about 1.75 to about $1.25 \mathrm{GPa}$ and the $a$-Se's reduced modulus by the same scheme drops from about 10 to about $6.5 \mathrm{GPa}$. The reduced modulus of PP calculated by the present method of correcting both $h_{\mathrm{c}}$ and $S$ for creep is in the range from about 1.75 to $2.0 \mathrm{GPa}$, while that of $a$-Se

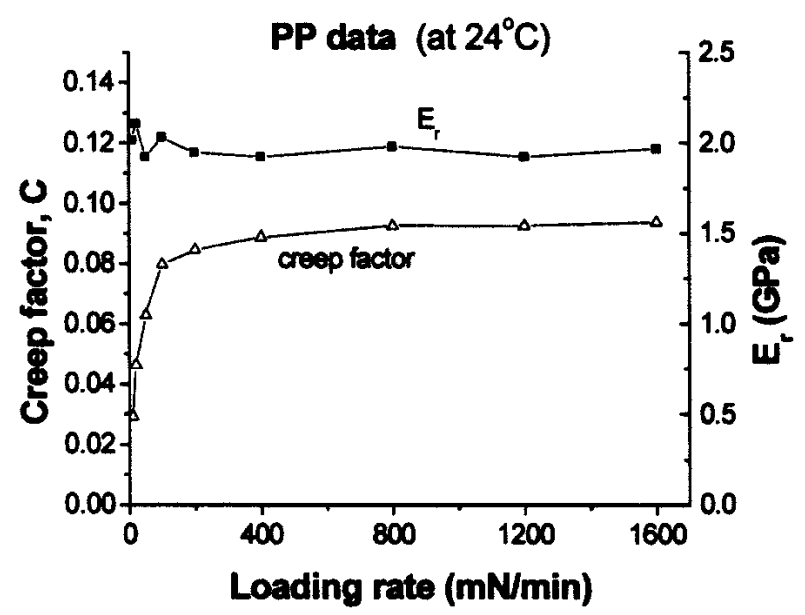

FIG. 12. Effect of loading rate on creep factor and creep-corrected reduced modulus in PP. All load schedules have maximum load $200 \mathrm{mN}$, holding time $60 \mathrm{~s}$, and unloading rate $400 \mathrm{mN} / \mathrm{min}$. 
is in the range from about 9 to $11 \mathrm{GPa}$. Both the PP's and the $a$-Se's modulus calculated by the present scheme do not have the trend to decrease with creep factor and agree well with the results calculated from Eq. (12) and Fig. 9, which have been described above.

Finally, it is worth mentioning that although the extent of creep at the point of unloading will depend on the load history beforehand, the applicability of the present creep correction procedure itself is not affected by the load history. The load history will affect the creep rate before unloading $\dot{h}_{\mathrm{h}}$ and the observed unloading stiffness $S$, but the applicability of the creep correction procedure itself [i.e., the validity of Eqs. (3), (7), and (8)] will not be affected. This can be seen from the results shown in Fig. 12 for a set of independent indentation experiments on PP. Here, the load schedules have different loading rates, but they all have the same maximum load, holding time at the peak load, and unloading rate. It can be seen that the creep factor is very small for small loading rates, but it quickly saturates to a constant value of about 0.09 as the loading rate increases. The reason is that much of the creep deformation would be completed during a slow loading, and hence, the creep factor, which is evaluated at the point of unloading [Eq. (14)], would become very small. Despite the fact that the creep factor at unloading a strong function of the loading rate when the latter is small, the creep-corrected reduced modulus is nearly constant for all loading rates as shown in Fig. 12. This indicates that the validity of the creep correction procedure itself is not affected by the loading rate.

\section{CONCLUSIONS}

Using polypropylene and amorphous selenium as prototype viscoelastic materials at room temperature, it is demonstrated that the original Oliver-Pharr method can give rise to unreliable results for the contact depth at the onset of unloading. A modified formula was proposed that can predict more satisfactorily the contact depth.
Together with the earlier method for creep correction for the contact stiffness, the present approach provides an effective procedure for measuring accurately the reduced modulus of a viscoelastic material by depth-sensing indentation.

\section{ACKNOWLEDGMENT}

The work described in this paper was partially supported by a grant from the Research Grants Council of the Hong Kong Special Administrative Region of China (Project No. HKU 7062/01E).

\section{REFERENCES}

1. W.C. Oliver and G.M. Pharr, J. Mater. Res. 7, 1564 (1992).

2. G.M. Pharr and A. Bolshakov, J. Mater. Res. 17, 2660 (2002).

3. G. Feng and A.H.W. Ngan, J. Mater. Res. 17, 660 (2002).

4. G. Feng and A.H.W. Ngan, in Fundamentals of Nanoindentation and Nanotribology II, edited by S.P. Baker, R.F. Cook, S.G. Corcoran, and N.R. Moody (Mater. Res. Soc. Symp. Proc. 649, Warrendale, PA, 2001), p. Q7.1.1.

5. M. Sakai and S. Shimizu, J. Non-Cryst. Solids 282, 236 (2001).

6. S.A. Syed Asif and J.B. Pethica, Philos. Mag. A 76, 1105 (1997).

7. B.D. Beake and G.J. Leggett, Polymer 43, 319 (2002).

8. T. Chudoba and F. Richter, Surf. Coat. Technol. 148, 191 (2001).

9. X. Li and B. Bhushan, Mater. Charact. 47, 1 (2002).

10. I.M. Low, Mater. Res. Bull. 33, 1753 (1998).

11. T.K. Harris, E.J. Brookes, and R. Daniel, Int. J. Refract. Met. Hard Mater. 17, 33 (1999).

12. S.A. Syed Asif and J.B. Pethica, in Thin Films: Stresses and Mechanical Properties VI, edited by W.W. Gerberich, H. Gao, J-E. Sundgren, and S.P. Baker (Mater. Res. Soc. Symp. Proc. 436, Pittsburgh, PA, 1996), p. 201.

13. A.A. Elmustafa and D.S. Stone, Acta Mater. 50, 3641 (2002).

14. M. Sakai, S. Shimizu, N. Miyajima, Y. Tanabe, and E. Yasuda, Carbon 39, 605 (2001).

15. B.N. Lucas and W.C. Oliver, Metall. Mater. Trans. A 30A, 601 (1999).

16. T.Y. Tsui and G.M. Pharr, J. Mater. Res. 14, 292 (1999).

17. A.H.W. Ngan and B. Tang, J. Mater. Res. 17, 2604 (2002).

18. I.N. Sneddon, Int. J. Eng. Sci. 3, 47 (1965).

19. S. Etienne, G. Guenin, and J. Perez, J. Phys. D 12, 2189 (1979).

20. M.F. Ashby and D.R.H. Jones, Engineering Materials (Pergamon Press, Oxford, U.K., 1986), p. 31.

21. R. Böhmer and C.A. Angell, Phys. Rev. B 48, 5857 (1993). 